\title{
The Traffic Capacity Variation of Urban Road Network due to the Policy of Unblocking Community
}

\author{
Xin Feng $\mathbb{D},{ }^{1,2}$ Yue Zhang $\mathbb{D},{ }^{3}$ Shuo Qian $\mathbb{D}^{4},{ }^{4}$ and Liming Sun $\mathbb{D}^{5}$ \\ ${ }^{1}$ School of Economics and Management, Yanshan University, Qinhuangdao 066004, China \\ ${ }^{2}$ School of Management, Hebei GEO University, Shijiazhuang 050031, China \\ ${ }^{3}$ School of Urban Geology and Engineering, Hebei GEO University, Shijiazhuang 050031, China \\ ${ }^{4}$ HuaXin College, Hebei GEO University, Shijiazhuang 050031, China \\ ${ }^{5}$ School of Languages and Culture, Hebei GEO University, Shijiazhuang 050031, China \\ Correspondence should be addressed to Liming Sun; sunlm1976@163.com
}

Received 6 May 2020; Revised 4 March 2021; Accepted 17 March 2021; Published 28 March 2021

Academic Editor: Lucia Valentina Gambuzza

Copyright $\odot 2021$ Xin Feng et al. This is an open access article distributed under the Creative Commons Attribution License, which permits unrestricted use, distribution, and reproduction in any medium, provided the original work is properly cited.

\begin{abstract}
At present, urban traffic congestion is a common problem of urban development in China. Therefore, China's government issued the policy of opening the gated communities in 2016, hoping to alleviate traffic pressure to some extent. But, at present, the quantitative empirical research on the effect of the policy implementation is less and more idealistic. In order to complete the leap from research on local isolated traffic capacity of static gated communities to research on global coupling traffic capacity of dynamic multilayer network and, to a certain extent, reflect the innovation of the research model and method, we make a quantitative analysis of the effect of the policy more consistent with the actual situation, which provides a quantitative management basis for the implementation of the policy. Based on literature review, this paper carried out two stages of research. Stage one consists of constructing traffic capacity assessment model-Road Capacity Assessment (RCA) model based on BPR (Bureau of Public Roads) impedance function from a simple local static perspective. Considering the factors such as delay of signalized intersection and travel time, through modeling analysis and empirical test, it is found that about $12 \%$ of local traffic capacity can be improved after open community. Stage two consists of constructing a global capacity model based on multilayer complex network coupling from the perspective of complex global dynamics. Considering the various network nodes of pedestrians, nonmotor vehicles, and other factors, we construct a multilayer complex network and dynamic superposition coupling. The empirical data show that the overall traffic capacity of the open community is improved by at least $11.3 \%$. Finally, it can be concluded that a significant increase in the global traffic capacity of complex urban road networks means that the losses caused by traffic congestion will be reduced. In some first-tier cities, the direct monthly income of the open-gated community is as high as 230 million yuan, not to mention its overall economic, social, and environmental benefits.
\end{abstract}

\section{Introduction}

1.1. Background. With the rapid development of China's economy, people's quality of life has been improved, the travel demand is also increasing, and the number of private cars is increasing. But, the corresponding road capacity has not been significantly improved, leading to serious traffic congestion.

By December 2018, the number of private cars in China has reached 206 million [1]. Therefore, the demand for urban traffic capacity also increases correspondingly. However, the existing urban traffic structure seriously restricts the development of traffic. Many scholars have conducted studies on this issue. Zhao [2] analyzed the causes of low road capacity by studying the current traffic situation in Hefei City, Anhui Province, in terms of the limitations of traffic planning, low road capacity, and weak awareness of traffic law. Taking Jinchang City in Gansu Province as an example, Yang [3] found that road traffic in small industrial cities suffers from insufficient urban road area, traffic infrastructure lagging behind the development of the city, imperfect road parking system, and slow development of public 
transportation. Tang and Zhang [4] also mentioned the problems of poor road infrastructure and backward traffic management, lack of facilities, and unsound institutions in road traffic of small towns, further illustrating the constraints of road structure on urban traffic development; meanwhile, Wu et al. [5] studied the effects of rectangular urban network aspect ratio on traffic system dynamics based on cellular automata modeling, and used Macroscopic Fundamental Diagram (MFD) to examine the performance of the road network, and the maximum arrival rate and critical congestion density of the MFD were studied as two main indicators of the system performance. It is found that the arrival rate of the square network is maximum under the closed boundary condition, but the congestion critical density is relatively low. As the aspect ratio increases, the arrival rate decreases and the critical congestion density increases. Cao et al. [6] conducted a multicause automatic real-time identification of traffic congestion on urban roads based on Bayesian networks. It was found that road access problems such as pedestrian influence, peak traffic, parking occupied road, unreasonable signal timing, and impact of traffic crossing the road are the main causes of traffic congestion. Various studies have shown that the road network structure has a great influence on the development of urban traffic to some extent. In the above studies, it can be shown that the road network structure greatly affects the development of urban traffic. The existence of gated communities is an important factor causing this result. It not only wastes effective road resources but also easily blocks the urban road network, which further aggravates the pressure of the urban road network [7].

Many developed countries, such as the United States, explored the opening of gated communities as early as the 1980s. After years of exploration and experiment in some cities, considerable experimental results have been achieved. For example, in Seaside town, Florida, which was listed by Time magazine as one of America's “Top Ten Design Achievements" for nearly a decade, the streets radiating from the center of the neighborhood are connected to the surrounding neighborhood, and the distance between the neighborhood and the center is controlled within a quarter of a mile, meaning that it takes only five minutes to walk from the neighborhood to the town center [8]. In France, Switzerland, Japan, and other countries, there are very few gated settlements to date. This is mainly because the development of gated settlements in each country is closely related to its own political, cultural, and economic background [9].

On February 21, 2016, to solve the above problems, the government once again elevated "urban construction" to the central level for specialized research and deployment. And, the State Council issued the relevant document "Several Opinions on Further Strengthening the Management of Urban Planning and Construction," which states in Article 16 on the promotion of the block system. "In principle no longer build gated communities. Those residential communities and unit compounds that have already been built should be gradually opened, internal roads should be publicized, traffic network layout problems should be solved, and land conservation and utilization should be promoted." The biggest benefit of promoting the urban block system is to solve the problem of traffic capacity, which is conducive to the formation of a road structure with narrow roads and dense road networks. If the urban network is extended in all directions, instead of just relying on a few main roads, the traffic congestion problem will be readily solved.

Based on the above background, the traditional gated community structure will no longer be applicable but will be replaced by an open community with more advanced concepts and more adapted to the requirements of modern urban development. This paper makes a quantitative analysis and evaluation of the influence of the gated community on the road capacity by constructing a mathematical model.

1.2. The Research. At present, in China, the construction of residential areas in each city is still based on a mainly gated community. Because the urban traffic flow has the characteristics of high density and strong continuity, gated communities not only waste effective road resources but also cause traffic jams in the city, resulting in low density of urban roads network, incomplete branch roads network, and overcrowded main roads.

At present, most papers mainly use cellular automation algorithm [10], topology probability analysis [11], fuzzy theory [12], and other mathematical methods. These kinds of methods can provide a large amount of experimental data at the present stage when open communities' samples are severely under-sampled. However, there are also some disadvantages such as lack of empirical evidence and low validity of conclusions.

The paper [13] used pedestrian area graphic analytic method, index method, and the transportation geography space syntax to analyze and compare the pedestrian system before and after opening closed communities in the Shanghai Taipingqiao area, and concluded that the gated communities have a large negative impact on the city's pedestrian transportation network but did not point out the impact on the overall transportation network. In the paper [14], the spatial syntax Depthmap software was used to analyze the enclosed residential areas within the selected range. The changes of road network in closed residential areas before, after, and in surrounding closed residential areas after opening are analyzed and compared, and the characteristics of road network in these three modes are summarized. He and Liu [15] used the road saturation degree at the macro level and the average speed of road network vehicle operation at the micro level as the judgment indexes to construct the evaluation model as well as the BPR function to optimize the Brees paradox model to analyze the range of reasonableness of opening the community, and then explore the impact of opening the gated community on the surrounding road traffic. The analysis shows that the selection of small-scale neighborhood opening, appropriate consideration of road network density increase, and consideration of selective opening of neighborhood roads are conducive to the development of urban traffic. Zheng and Wang [16] compared two construction modes of gated and 
open communities from the perspective of sustainable urban development and concluded that open communities are more effective in terms of land resource utilization, reducing pollutant emissions, protecting the ecological environment, relieving urban traffic pressure, and promoting social harmony, and are more in line with the current concept of sustainable development, which can be considered under the premise of the actual situation in different areas. The open residential area can be gradually promoted, taking into account the actual situation of different regions. Based on the analysis of the whole urban road network, this paper studies the integration degree and depth of the gated community and explores the influence of different open modes on the urban road network. The study in [17] proposes a long-term forecasting scheme and implementation method based on the interval type-2 fuzzy sets theory for traffic flow data.

Given the situation described in the previous paragraph, this paper draws on a large number of different research literature to develop the research idea and methodology of "prediction first, simulation later, verification" in the phase I research. The results of the study were first predicted, and then, by selecting two indicators, namely, signal intersection delay and travel time, and combining them with the BPR obstruction function, a model was constructed to evaluate the impact of opening the communities on the road capacity, and the data obtained from traffic simulation were used to quantitatively analyze the degree of impact of opening different types of communities on the surrounding roads. To improve the validity of the conclusion, the model is further verified empirically, and the conclusion is reached on the validation results. In the study of stage 2 , the static gated communities in stage 1 are taken as the node, the road is taken as the edge, and the characteristics of mobile and nonmobile traffic units are fully considered to construct the multi-layered dynamic complex network, and the coupling analysis and network topology analysis of the complex network are carried out by using the relevant methods of the complex network to obtain the corresponding conclusion.

In general, mathematical modeling and various methods in complex networks are comprehensively applied to complete the construction of systematic research ideas and the selection of research methods from static to dynamic and from local to overall.

\section{Analysis of Urban Traffic Structure in the Presence of Gated Communities}

2.1. Analysis of the Current Status of the Urban Road Network. Compared with the urban road structure of Western developed countries, the current density of China's road network is seriously inadequate, and the main roads are responsible for the main traffic functions. This situation leads to poor superposition, low shunt capacity, and low organic dispersion capacity between roads. The main reason for this situation is the existing community structure. China's current Planning Standards for Comprehensive Urban Transportation System is a national standard, which clearly stipulates the density of the road network at various levels in cities. Influenced by the early theories of rural cities and neighborhood units, urban traffic abroad tends to be safer, more convenient, and less polluting. In the design of the urban road network, it pays attention to the coordination of roads at all levels, and the functions of roads at different levels are clear. In terms of the ability of diversion and evacuation, it is far superior to domestic traffic [18].

Compared with foreign road networks, it can be seen that the density of China's urban road network is far lower than that of foreign countries. At present, domestic urban planning does not attach importance to the planning and construction of urban branch road networks but only focuses on the main roads of each city, which further aggravates the situation of insufficient density of the overall road network.

2.2. Types of Gated Community Structure. At present, the road traffic organization mode in the residential district of China can be summed up as three types according to the nature of traffic: pedestrian-vehicle sharing, pedestrian-vehicle incomplete diversion, pedestrian-vehicle diversion. The disadvantages that lead to their existence also vary due to the same functions assumed and the condition of the roads.

2.2.1. Pedestrian-Vehicle Sharing Structure. The pedestrianvehicle sharing modes are mostly based on the graded road network, and the roads in residential areas can be divided into three grades: trunk road, branch road, and secondary trunk road. The trunk road indicates that the main trunk road inside the residential area mostly plays the role of connecting the exits and entrances in the residential area, the vehicle traffic rate is the highest among the three grades of roads, and the traffic flow is the largest, but because the residential area is not open to the public, the traffic resources of the trunk road are wasted. The branch road refers to the road between the modules in the residential area. Its traffic speed and its traffic flow are lower than the trunk roads. The data from all aspects of the secondary trunk road are the lowest, which mainly plays the role of traffic evacuation. Traffic accidents occur frequently because pedestrians and cars are not diverted from branch roads and secondary trunk roads.

2.2.2. Pedestrian-Vehicle Incomplete Diversion Structure. The pedestrian-vehicle incomplete diversion mode indicates the partial separation of motorized, nonmotorized, and pedestrian traffic on residential roads by the installation of special roads in the internal road composition of residential areas. The use of green belts, for example, separates motorized roads from sidewalks, creating localized, dedicated walking paths. This mode of diversion improves pedestrian safety within residential areas to a certain extent and can assume the function of the motor vehicle lane to a certain extent. However, the main road under this structure is still used only by people in the community, and it cannot share the traffic flow of urban roads and still not improve the current situation of urban road congestion. 
2.2.3. Pedestrian-Vehicles Diversion Structure. The pedestrian-vehicle diversion mode indicates that through the special classification of the inner road of the district, the special road system with different functions is established, so that all kinds of traffic operation modes do not interfere with each other, to realize the purpose of the passenger-vehicle diversion. Although this structure can reduce the occurrence of traffic accidents, the increase in investment has increased the burden of the social economy, and the road-use rate has not been improved accordingly.

At this stage, most of China's closed communities use a graded road network (i.e., pedestrian-vehicle sharing) to form the typical road structure in residential areas. With the increase of car ownership in urban residential areas in China, the traditional road system design scheme in residential areas is no longer suitable for its traffic development, and the phenomenon of internal road congestion appears frequently.

2.3. Study and Discussion on the Improvement of Road Network Capacity by Opening Communities. At present, traffic congestion is a common problem in cities at all levels, especially in special periods and places. Through investigation and research, in this paper, it is found that the impact of the opening of closed communities on improving road capacity can be summarized in the following three ways:

(1) Share the main flow: due to the excessive length of some communities, the bypass distance of the surrounding roads has increased and the traffic volume is concentrated, which leads to the superimposition of flow and increases the pressure on urban traffic. After opening, the superimposed flow in the main road can be traversed through the residential area, sharing the traffic pressure on the main road increasingly.

(2) Alleviate the accumulation of human traffic at a point: during the rush hour, there is a large flow of people at the entrance and exit of the community, which increases the bearing pressure of the road. Meanwhile, various uncertainties affect the traffic increase, which further affects the capacity of the road. After the opening, the community became many small housing groups, and the flow of people from the original main entrance of the community dispersed to the groups, avoiding the accumulation of a large number of people flow at a certain point.

(3) Enrich the network structure: generally, the residential communities are located near the main roads with convenient transportation. While surrounded by the main roads, they also cut off the branches at all levels attached to the main roads, reducing the selectivity of traffic and reducing the capacity of traffic. After the opening, the internal road network of the community is integrated into the urban road network system, which makes the branch road network of the urban road network richer and more accessible.
On the whole, after the opening of the existing gated community, the network structure of adjacent roads will be enriched, traffic pressure will be alleviated, and the micro-circulation system in urban roads will be increased, so that the internal roads of the community and urban roads will form an organic whole. When vehicles move to the road near the open community, more routing options will be available, which greatly disperses the flow on the main road and reduces the traffic congestion caused by the flow backlog.

2.4. Research on Conflicts and Interests under Nongated Communities. Nongated communities can further improve the efficiency of traffic, and at the same time, the safety of pedestrians will become a topic of concern. Traffic accidents in the communities may increase, and in the light of the actual situation of traffic management in China, in a way, perhaps the opening of the gated communities will instead help to solve the problem of safety as well as the conflict of public interests [19]. There are three main aspects:

(1) After the gated communities are opened, the roads in the communities are already equivalent to the roads in the general sense to some extent, so their management will also be included in the management of ordinary roads and the corresponding laws and regulations will be implemented. To further strengthen people's awareness of road traffic laws and regulations, people will pay more attention to driving safety on ordinary roads compared to what was previously thought to be roads within the communities, because they have been included in the scope of road laws and regulations management in the usual sense. The corresponding punishment measures will be more strictly enforced.

(2) The roads of the communities after opening are subordinate to the municipal network, so the corresponding supporting facilities, such as traffic lights, cameras, etc., will be further standardized and improved, which will also help supervise the traffic safety in the communities. At the same time, it also helps residents to develop a good habit of observing traffic rules as soon as they go out, instead of thinking that they can be more relaxed in the communities.

(3) With the gradual implementation of smart municipal projects in smart cities such as smart transportation and smart road networks, after the opening of the community, the management level of public areas will be further improved. Real-time guidance of traffic flow, dynamic regulation of parking spaces, also known as shared parking spaces [20], and timely investigation and tracking of traffic accidents can be achieved, which will greatly enhance rather than weaken the safety of residents, and also further improve the optimal allocation of public resources.

With the opening of the community and the improvement of traffic efficiency, people's awareness in terms of safety and law is also increasing in parallel, and the relevant departments will further strengthen the management level of 
other aspects at the same time. On the whole, the increase of community road network capacity is conducive to the optimization of multifaceted resource allocation and the promotion of social management construction.

\section{Research on Local Isolated Traffic Capacity in the Static Gated Communities}

Based on the research and analysis of the current urban structure and the structure of the gated community itself, this paper selects the delay and travel time of the signalized intersection as two indexes, combines with the BPR impedance function, and constructs the evaluation model of the influence of the opening of the community on the road capacity-the RCA model, to quantitatively analyze the impact of different types of closed communities on the surrounding roads and empirically verify the model.

3.1. Establishment of Road Capacity Assessment Mode [21]. The basic traffic capacity of urban road refers to the maximum number $N$ of vehicles passing through a section of a road in unit time under ideal conditions when standard vehicles travel continuously at a minimum headway interval of $l_{0}$ :

$$
\begin{aligned}
& N=\frac{3600}{t_{0}}=\frac{3600}{l_{0} /(v / 3.6)}=\frac{1000 v}{l_{0}} \\
& l_{0}=l_{1}+l_{2}+l_{3}+l_{4} .
\end{aligned}
$$

Here, $l_{1}$ means the travel distance that the vehicle travels within the reaction time of the driver when braking; $l_{2}$ refers to the distance that the vehicle travels from braking to a complete stop when braking; $l_{3}$ refers to the safety distance between two vehicles; $l_{4}$ refers to the standard vehicle length.

$$
\begin{aligned}
& l_{1}=\frac{v}{3.6} t, \\
& l_{2}=\frac{v^{2}}{254 \cdot \varphi} .
\end{aligned}
$$

According to formulae (3) and (4), the formula of the minimum headway interval between vehicles can be converted:

$$
\begin{aligned}
& l_{0}=l_{1}+l_{2}+l_{3}+l_{4}=\frac{v}{3.6} t+\frac{v^{2}}{254 \varphi}+l_{3}+l_{4,}, \\
& C=N \varepsilon_{1} \varepsilon_{2}, \ldots, \varepsilon_{n} .
\end{aligned}
$$

In formula (5), $\varphi$ means the longitudinal adhesion coefficient of the tire, which is related to tire pattern, road roughness, vehicle speed, and other factors. $t$ is the driver's response time. According to relevant literature, this variable is generally taken as $2 \mathrm{~s}$. In the actual situation, the capacity of the road is affected by many uncertain factors, such as road construction, pedestrian crossing, and so on. In the face of the opening community, many influencing factors interfere with each other. In order to make the model more accurate, the road correction coefficient is introduced, and the capacity of a certain road section can be expressed in formula (5) (where $\varepsilon_{1}, \varepsilon_{2}, \ldots, \varepsilon_{n}$ are the correction coefficients of each factor).

3.2. Analysis of the Topology Structure of the Road Network inside the Community. The road conditions in different sections of the road in the community are also different. Different road traffic capacity correction models will be developed below for the three prevalent no-signal intersections, loop intersections, and signalized intersections to make the final ratings more accurate.

3.2.1. Unsignalized Intersections. According to the clearance interlude theory, in the unsignalized intersection, the number of gaps that can be crossed by the traffic flow in the priority direction is calculated directly, which is the maximum traffic volume that can be passed through in the nonpriority direction. The traffic flow on the main road makes it a continuous traffic flow. According to the references, the probability distribution of vehicle arrival is Poisson distribution, and the time interval distribution between vehicles is the negative exponential distribution.

First, at the cross intersection, when the insertable interval is $\alpha$, the traffic flow on the branch road can pass through the following sight distance of $\beta$, then the calculation formula can be obtained as follows:

$$
Q_{b}=\frac{Q e^{-\lambda \alpha}}{1-e^{-\lambda \beta}} .
$$

Here, $Q_{b}$ is the traffic flow that can be passed in the secondary road; the $Q$ is traffic flow in the main road $\lambda=\alpha / 3600$; and $\alpha$ means the critical interval time.

Second, T-junction, $Q_{12}$ means traffic capacity of return right nonpriority of nonpriority direction, $Q_{13}$ means traffic capacity of return left nonpriority of nonpriority direction.

$$
\begin{aligned}
Q_{12} & =\frac{Q_{32}\left(1-\beta_{1} Q_{32}\right)}{e^{\left(\alpha-\beta_{1}\right) Q_{32}}\left(1-e^{\beta_{2} Q_{32}}\right)}, \\
Q_{13} & =\frac{\left(Q_{32}+Q_{23}\right)\left(1-\beta Q_{32}\right)\left(1-\beta_{1} Q_{23}\right)}{e^{\left(\alpha-\beta_{1}\right)\left(Q_{32}-Q_{23}\right)}\left(1-e^{-\beta_{2}\left(Q_{32}+Q_{23}\right)}\right)},
\end{aligned}
$$

3.2.2. Roundabout Intersection. Roundabout refers to the setting of traffic island at the center of the intersection, which is convenient for vehicles entering the intersection to detour in the same direction. This avoids direct crossovers, collisions, and large angle collisions, utilizing the Waldrop formula:

$$
Q=354 W \frac{(1+(e / W))(1-(p / 3))}{1+(W / l)} .
$$

Here, $Q$ means the maximum traffic capacity in the weaving section; $W$ means the width of the road in the weaving section; $e=\left(\left(e_{1}+e_{2}\right) / 2\right)$ means the average length 
of the roundabout intersection entrance site; $P$ means the proportion of vehicles in total vehicles in the weaving section; and $l$ means the length of the weaving section. According to the references and the actual situation, the design traffic capacity should be $80 \%$ of the maximum value of this formula actually. So this formula can be modified to

$$
Q=280 W \frac{(1+(e / W))(1-(P / 3))}{1+(W / l)} .
$$

In the process of calculation, it combined the open community. All vehicles are replaced by cars. The conversion coefficient of a car is $l$. The conversion coefficient of midsize vehicles is 1.5 . The conversion coefficient of large vehicles is 2. The conversion coefficient of oversize vehicles is 3.5.

3.2.3. Signalized Intersections. In signalized intersections, the traffic capacity of the road means the maximum number of vehicles passing the stop at each unit time in a certain phase. $T$ means cycles of signal light change. The passage time in a certain phase is recorded as $t_{g}$. The time of first vehicles passing the stop line is recorded as $t_{0}$. The index of vehicle pass intersection is reduced coefficient $\varphi$. The calculation formula can be expressed as

$$
Q=\varphi \frac{3600}{T}\left(\frac{t_{g}-t_{0}}{t_{s}}-1\right) .
$$

3.3. RCA Traffic Capacity Evaluation Model. To ensure the rationality of the evaluation system, this paper chooses two indexes: signalized intersection and travel time. Among them, the signalized intersection can reflect the surrounding road operation conditions, and the formation time is based on the U.S. Federal Highway Administration road resistance function (BPR function), which determines the road travel time and the relationship between the road section travel time and traffic volume. Combined with the interference correction factors of nonmotor vehicles and pedestrians, the average delay calculation formula of the final signalized intersection is

$$
d=\frac{0.5\left(1-\left(t_{g} / T\right)\right)}{1-\left[\min (1, x)\left(t_{g} / T\right)\right]},
$$

In the formula, $T$ indicates the period length of the signal; $t_{g}$ indicates the effective duration of the green light; and $x$ indicates the lanes' saturation. The lower the calculation result of this formula is, the better the running state of the road is; otherwise, the worse it is.

Based on the actual situation and the BPR functions, the travel time model is established as follows:

$$
\begin{aligned}
& t=t_{0}+\delta t_{0}\left(\frac{v}{C}\right)^{\gamma} q, \\
& t=t_{0}+\left[1+a_{1}\left(\frac{q}{Q}\right)^{\beta_{1}}+a_{2}\left(\frac{q_{b}}{Q_{b}}\right)^{\beta_{2}}\right] .
\end{aligned}
$$

Here, $\delta$ and $\gamma$ are taken as 0.15 and 4 , respectively. $Q$ means motor vehicles' vehicle flow of the road, $Q_{b}$ means design capacity nonmotor lane of the road, and other variables are undetermined parameters. Besides, according to pedestrians on road traffic correction coefficient (the result in Table 1) and interference coefficient nonmotor vehicles on motor vehicles,

$$
\begin{aligned}
& \varepsilon_{b}=0.8-\frac{\left(\left(q_{b} / Q_{b}\right)+0.5-f_{2}\right)}{f_{1}}, \\
& \mathrm{t}=\left\{\begin{array}{l}
t_{0}+\delta t_{0}\left(\frac{v}{0.8\left(0.8-\left(\left(\left(q_{b} / Q_{b}\right)+0.5-f_{2}\right) / f_{1}\right)\right) C}\right)^{\gamma} q+\frac{0.5\left(1-\left(t_{g} / T\right)\right)}{1-\left(\min (1, x)\left(t_{g} / T\right)\right)}, \\
t_{0}+\delta t_{0}\left(\frac{0.8\left(0.8-\left(\left(\left(q_{b} / Q_{b}\right)+0.5-f_{2}\right) / f_{1}\right)\right) v}{0.8 C}\right)^{\gamma} q+\frac{0.5\left(1-\left(t_{g} / T\right)\right)}{1-\left(\min (1, x)\left(t_{g} / T\right)\right)},
\end{array}\right.
\end{aligned}
$$

Here, $f_{1}$ and $f_{2}$ means single nonmotor and maneuver lane width, respectively. Formula (17) is the final evaluation model. The smaller the data obtained by $t$ are, the higher the capacity is.

According to the model, the simulation data obtained in conjunction with reality were brought into the model for calculation (see Table 2 for the results). Through the analysis, it is found that the opening of the community has a significant contribution to the traffic capacity of the surrounding roads, with a contribution of about 12 percent. During the calculations, it is found that the opening of some of the complex communities with the internal road network had a negative effect on the surrounding road traffic. So, not all gated communities can improve the road capacity of the surrounding area when opened. As a whole, opening the gated community can increase road network density and relieve traffic pressure of the main track and, meanwhile, increase road network intersection quantity and reduce the average speed of driving road. In general, opening the gated community is good for traffic capacity, but some communities are not suitable for opening, which in turn will reduce the traffic capacity. 
Table 1: Pedestrian interference correction coefficient.

\begin{tabular}{lcccccc}
\hline Disturbance degree & Very serious & Serious & Relatively serious & Common & Very small & No \\
\hline$\varepsilon$ & 0.5 & 0.6 & 0.7 & 0.8 & 0.9 & 1 \\
\hline
\end{tabular}

TABLE 2: The influence of the opening of the community on the traffic capacity of surrounding roads.

\begin{tabular}{lccccc}
\hline Community numbering & $\mathrm{A}(\%)$ & $\mathrm{B}(\%)$ & $\mathrm{C}(\%)$ & $\mathrm{D}(\%)$ & $\mathrm{E}(\%)$ \\
\hline Influence degree & 11.93 & -3.37 & 11.88 & 12.14 & 12.68 \\
\hline
\end{tabular}

3.4. The Empirical Analysis of Traffic Capacity: A Case Study of Songbai Community in Xiamen City. By querying the data of the National Bureau of Statistics and major traffic information websites, and combining the database data of CNKI, we can find and analyze the existing communities that meet the conditions. The Songbai community in Xiamen city has experienced the process of changing from a gated community to a semi-open community, which has better comparison data before and after, and can more accurately reflect the impact of the opening of the gated community, so it is selected as an example for research. The main data for evaluating the traffic capacity of the roads around the Songbai community are shown in Table 3 .

Based on the data obtained, the combined value of the road capacity before and after the opening of Songbai community can be obtained from models (1), (2), (5) and the corrected model (17) after taking into account the correction factors (Table 4), where different numbers represent different locations of the community. Due to the differences in the number of curves and road width contained in the different locations from 1-5, the final data obtained are quite different. Position 1 in the division is in the deepest part of the community, and external vehicle traffic is less, resulting in no significant change in the road capacity before and after the opening. Position 6 contains many curves. When vehicles travel to this position, the average speed has been affected, resulting in a decrease in traffic capacity compared with the preopening period.

Except the special positions of No. 1 and No. 6 locations, the average increase ratio in the capacity of the remaining locations before and after the opening of the community is $12.2 \%$, which is consistent with the conclusion of the simulation of $12 \%$. Position 6 can represent the complex internal road conditions, more curves of the community; position 1 is in line with the characteristics of the remote community, both verify the conclusion that "not all community opening can improve the ability of the surrounding road capacity." The model's findings have been verified so far.

\section{Study on the Global Coupling Traffic Capacity of Dynamic Multilayer Road Network}

To enhance the practical application of the project, the research object is expanded from a local road near a single residential community to the road network of the urban area, and Beijing, which has a severe traffic situation, is selected as the research object. This paper takes the districts of Dongcheng, Chaoyang, Haidian, and Fengtai, which are located within the Fifth Ring Road of Beijing, as the entry points. The region is regarded as connected graphs; all communities in the region are abstracted as joints, and roads are abstracted as paths.

\subsection{The Constructive Basis for Multilayer Complex Traffic Network}

4.1.1. The Evaluation Basis for a Single-Layer Traffic Network. In this study, the average shortest path, the maximum flow in the traffic network and Geordie Coefficient of network traffic distribution are taken as evaluation indexes.

(1) The average shortest path of the network. The shortest path between two nodes in a traffic network is the shortest distance between any two nodes. Then, the average shortest path is the average of the shortest distances of all node pairs. It is the most important parameter in traffic network research that can be used to evaluate the accessibility of the traffic network. The calculation formula is

$$
l=\sum_{i \neq j} D_{i j} l_{i j}
$$

In the formula, $l=\sum_{i \neq j} D_{i j} l_{i j}=1, D_{i j}$ means path traffic flow from node $i$ to node $j$. $l_{i j}$ means the shortest path length from node $i$ to node $j$.

(2) The maximum flow in the traffic network. The maximum flow in the traffic network represents the maximum volume of traffic in that network and can be used as a core indicator to evaluate the network's capacity to travel. In this study, the maximum flow is the core of the study and the Edmond-Karp algorithm is used to perform the study calculation.

(3) Gini coefficient of network traffic distribution. Gini coefficient is proposed by Italian economist Gini, which is used to evaluate whether the income distribution is even or not. Its value is between 0 and 1 . The closer the Gini coefficient is to 0 , the more evenly the income distribution is; the closer the Gini coefficient is to 1, the greater the gap between the rich and the poor is. Gini coefficient in economics is introduced to analyze the distribution characteristics of network traffic, which can more accurately 
TABLE 3: Main parameter data of Xiamen Songbai community before and after opening.

\begin{tabular}{lcccc}
\hline Index & The density of road network & Average road width & Traffic density & Cycle of stoplight \\
\hline Before opening & 7.37 & 20 & 220 & 111 \\
After opening & 9.34 & 15 & 150 & 126 \\
\hline Index & Green light time & Average speed of vehicle head & Average speed of vehicle \\
\hline Before opening & 48 & 5.18 & 60 \\
After opening & 54 & 4.16 & 45 \\
\hline
\end{tabular}

TABLE 4: Comprehensive evaluation of traffic capacity of surrounding roads before and after the opening of Songbai community in Xiamen city.

\begin{tabular}{lcccccc}
\hline Numbering & 1 & 2 & 3 & 4 & 5 & 6 \\
\hline Before opening & 0.500 & 0.466 & 0.480 & 0.427 & 0.447 & 0.501 \\
After opening & 0.500 & 0.521 & 0.485 & 0.517 & 0.514 & 0.500 \\
Improve proportion & 0.000 & 0.118 & 0.010 & 0.210 & 0.149 & -0.001 \\
\hline
\end{tabular}

evaluate whether the distribution of network traffic is even. If all traffic flows are distributed on a specific edge of the network, then the Gini coefficient used to describe the traffic flow distribution is 1 ; if the traffic flow distribution in the network is even, then the Gini coefficient approaches 0. Combined with the calculation method of Gini coefficient in economics, the Gini coefficient of traffic network flow distribution is defined as

$$
G=\frac{1}{2 E^{2} \bar{s}} \sum\left|s_{p}-s_{q}\right| .
$$

In the formulation, $E$ means the sum of the number of edges in the traffic network. $s_{i}$ denotes the flow of traffic passing boundary $i . \bar{s}$ is the average value of the total connected traffic flow in the network, $\bar{s}=\sum_{i} s_{i} / N$ (where $n$ is the total number of nodes in the network). Under the condition that the network traffic flow is evenly distributed, the traffic flows $s_{i}$ passing through link $i$ is linearly related to the number of shortest paths passing through link $i$. From the above calculation formula, it can be concluded that the value range of the Gini coefficient of traffic network flow distribution is [0, 1]. According to the definition of the Gini coefficient, the larger the Gini coefficient of network flow distribution is, the more uneven flow distribution in the traffic network is, and the more likely road traffic congestion will occur.

4.1.2. The Basis for Actual Road Network Construction. This paper refers to the state standard announced by the Ministry of Construction Standard of Urban Residential Areas Planning \& Design "(hereinafter referred to as Design Standard)." Article 8 of the Standard, the internal roads of a residential area can be divided into four levels: residential area roads, community roads, group roads, and inter-house roads.

In the period 1994-2002, the Design Standard was implemented at that time, which stipulated that the width of the road should comply: Community road: the road width is 5-8 meters; Group road: the road width is 3-5 meters; and House path: the width of the road should not be less than 2.5 meters. According to the Design Standard that was revised the latest in 2002, it stipulated that the pavement width of the community road should be 6-9 meters for the community built after 2002, and other parameters should remain unchanged. Through the study, it was found that the roads in the community before 2002 were not suitable for motor vehicles due to the narrow road width and poor road capacity after a long service life. At this time, only the influence of opening on pedestrian and nonmotor vehicle road capacity was considered. The road width of the community built after 2002 met the vehicular traffic standards, so both pedestrians and nonmotor vehicles, as well as the traffic capacity of motor vehicles, should be considered.

We select and investigate the road conditions of four communities in the districts of Chaoyang, Fengtai, Dongcheng, and Haidian, and Chaoyang constituted the main data. Fifty-one communities such as the Guoao-Cun Community and Meijing Dongfang Community are classified and data integrated. According to the data, there are 10 residential areas built before 2002, including Dianzicheng Community, Huating Community, WangjingHuayuanXiqu Community, Huizhongli Community, Shian-Jiayuan Community, Jiulong-Huanyuan Community, GuanzhuangXili Community, Nanhudongyuan first Community. And, all of the roads in these communities are four-level roads. But, in the communities built after 2002, four-level roads are distributed.

\subsection{Construction of the Traffic Network Diagram}

4.2.1. Construction of the Single Vehicle Traffic Network. Chaoyang District road network is regarded as the connected graph of the traffic network; all communities in the area are regarded as nodes, and all roads are regarded as paths. According to the Provisional Provisions on Urban Planning Quotas and Indicators issued by the National Construction Commission, the maximum road width of different grades of roads is used as a measure of the maximum capacity of each path, and each path is recorded as a unit capacity to obtain the data of each grade of roads in accordance with the classification of roads in the Planning Standard of Comprehensive Urban Transportation System (as shown in Table 5) [22]. 
TABLE 5: Each level of road corresponding to the road width and maximum capacity table.

\begin{tabular}{lcccc}
\hline Road level & Level 1 & Level 2 & Leve 3 & Level 4 \\
\hline $\begin{array}{l}\text { Width of } \\
\text { motor vehicle } \\
\text { road }\end{array}$ & $3.5 \sim 3.75$ & $3.25 \sim 3.5$ & $3.25 \sim 3.5$ & $3.25 \sim 3.5$ \\
\hline $\begin{array}{l}\text { Number of } \\
\text { one-way traffic } \\
\text { lanes }\end{array}$ & $\geq 4$ & $\geq 4$ & $\geq 2$ & $\geq 2$ \\
$\begin{array}{l}\text { Overall road } \\
\text { width }\end{array}$ & $40 \sim 70$ & $30 \sim 60$ & $20 \sim 40$ & $16 \sim 30$ \\
\hline
\end{tabular}

According to the above standard abstraction for transportation network connected graph, 51 residential communities and main roads are connected in the Chaoyang district, because the district construction road conditions were poor before 2002, inappropriate to traffic after opening, so in the construction of motor vehicle traffic network, only the communities built after 2002 are considered. The schematic map of the distribution (Figure 1) and network of the Chaoyang residential district (Figure 2) is as follows.

According to the ratio $k$ between the actual traffic flow and the maximum traffic flow of a concrete road, the path in the corresponding network diagram of the road is assigned. The actual flow $G$ is $P_{\max } * k$. In terms of Beijing, $k$ value ranges from 0.8 to 1 . According to this method, each path in the traffic network connectivity map is assigned to obtain the motor vehicle traffic network map.

Because of the different locations and self-structure of each district, the impact of opening on road capacity is also different. According to the prophase simulation data and related literature data, first of all, a normal distribution with $12 \%$ as the expectation is made for its influence degree. MATLAB software program is used to generate a random number, and 51 random numbers are used as the influence rate of 51 residential districts in the Chaoyang district. Secondly, according to the standards of the roads corresponding to the maximum capacity value at all levels and the rate of actual traffic flow to the maximum design flow, the maximum capacity of each path is obtained together with the existing flow value $G$, and the latest flow value of each path is calculated together with the impact ratio after opening. Finally, the Edmond-Karp algorithm is used to compare the maximum flow calculations of the connected road network with different flow values before and after opening gated communities. Combined with the average shortest path of the network and the Gini distribution coefficient of traffic flow and other parameters, the calculation results show that the opening of closed communities improves the traffic capacity of road vehicles by $7.2 \%$.

\subsubsection{Construction of Single-Layer Nonmotor Vehicle Traffic} Network. The opening of gated communities can not only improve the capacity of road vehicles but also improve the capacity of pedestrians and nonmotorized vehicles. Referring to available literature, we have obtained that the relationship between the capacity of nonmotorized vehicles in a traffic network and the capacity of motor vehicles on this section of the road is

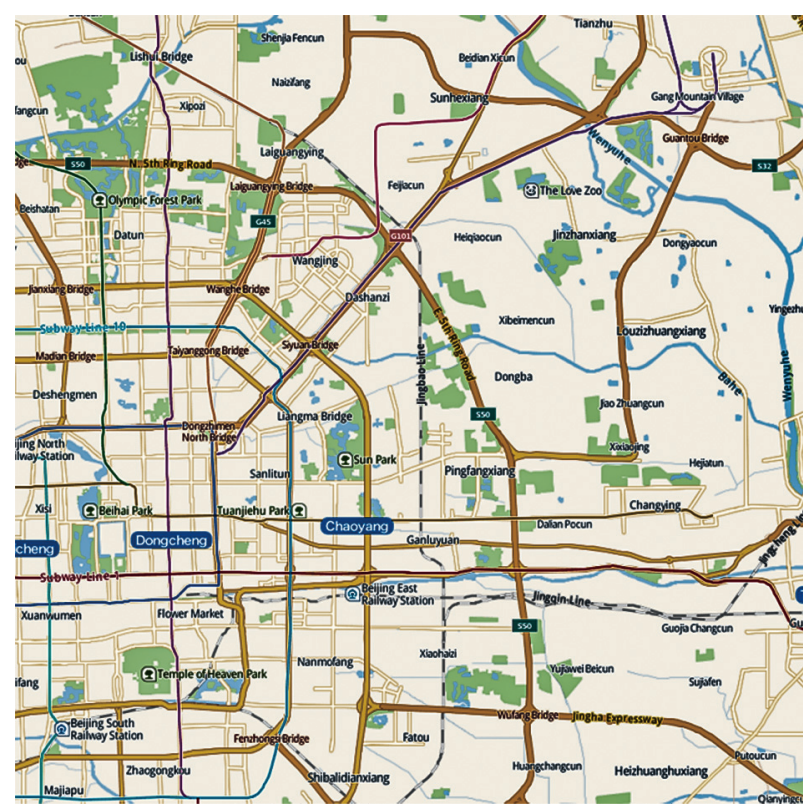

Figure 1: A distribution map of residential areas in Beijing's Chaoyang district.

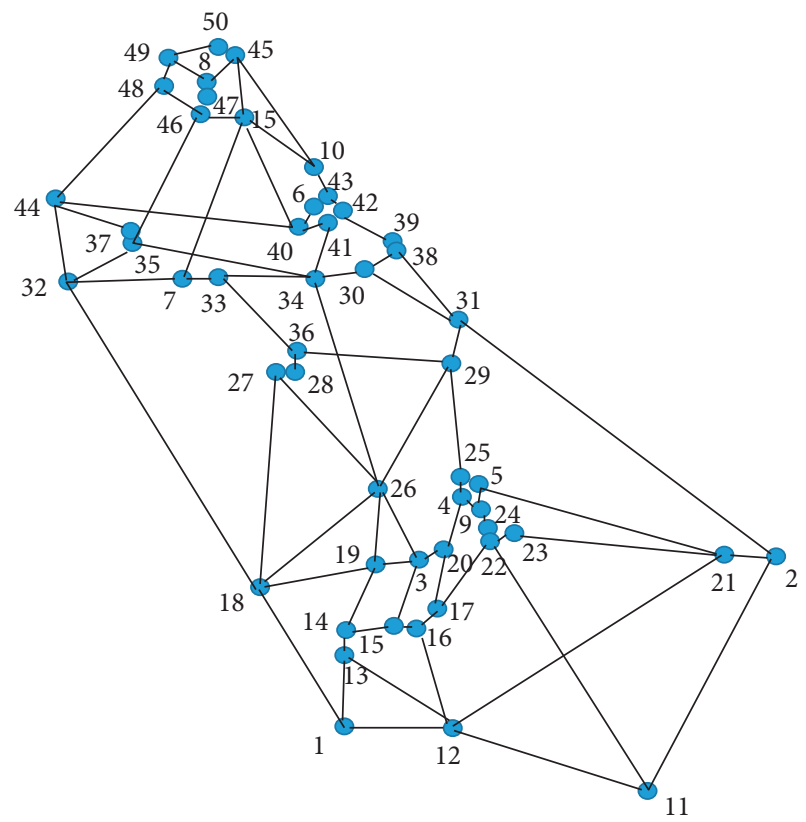

FIgURE 2: Traffic network diagram.

$$
N_{p} \cdot \lambda_{a}=N_{d},
$$

where $\lambda_{a}$ is the equilibrium parameter introduced to correspond the capacity of nonmotor vehicles to the capacity of motor vehicles. According to the available literature in the study of the frontline city network, the value of $\lambda_{a}$ is 0.4 , which is more reasonable.

Considering that there are many similarities between pedestrian and nonmotor vehicle traffic status and motor vehicle traffic status, the motor vehicle capacity evaluation model is used to evaluate the nonmotor vehicle traffic status. 
According to the characteristics of slow passage speed and flexible passage path of pedestrians and nonmotor vehicles, the parameters of the original model are specially treated in accordance with the characteristics of pedestrians and nonmotor vehicles.

The parameters are substituted into the model used to calculate the capacity of pedestrians and nonmotor vehicles (17). Combined with the models (17) and (20), it can be calculated that when the road vehicle capacity increases by $12 \%$ after the gated community is opened, the ratio of nonmotor vehicle capacity increases by $29 \%$.

As the improvement of pedestrian capacity is not much affected by the construction period of the residential life, all communities in the selected area are taken as nodes to construct the nonmotor vehicle traffic network, and the method used in constructing the motor vehicle traffic network is applied to assign a value to the nonmotor vehicle traffic network for each path in the vehicle traffic network.

\subsection{Construction of Dynamic Multilayer Network.} Considering the interaction between motor vehicle network and nonmotorized network, and considering the influence of the change of one node flow state of a single layer network on the state of other nodes of the network and each node of another network, the superimposed coupling of motor vehicle and nonmotor vehicle network is carried out, and the final degree of promotion is calculated.

Combined with the above research, the theoretical capacity of motor vehicles $N_{d}$ can be obtained. The capacity of nonmotor vehicles is $N_{p}$. Referring to the existing literature, we build the final capacity calculation model of the road:

$$
N^{\prime}=\mu \cdot N_{d}+v \cdot N_{p}
$$

After considering pedestrian, nonmotor vehicle, and other interference factors, the numerical value fluctuates between 0.65 and 0.90 according to the different road conditions. Combined with the actual road conditions of the studied area, the value is 0.7 , that is, the actual capacity of road motor vehicles is 0.7 times of the theoretical value. It is a coefficient introduced to match the capacity of nonmotor vehicles and motor vehicles. According to the previous studies, the values fluctuate between 0.1 and 0.4, depending on different road conditions.

The values of each parameter are brought into the model to calculate the comprehensive capacity of the urban road network. Compared with the values obtained before and after the opening of the district, the following conclusion can be drawn: the opening of the gated communities can improve the traffic capacity of the urban global road network by at least $11.3 \%$. Compared with other studies, although $11.3 \%$ seems slightly lower, this study modifies the problem of repeated calculation of traffic flow data at intersections in previous research methods, and comprehensively considers the influence of pedestrians and nonmotor vehicles on motor vehicle traffic from the perspective of multilayer complex network coupling. On this basis, the conclusion of the global traffic capacity of the road network is more in line with reality than the previous research.

\section{Application and Effect Evaluation of Research Results}

5.1. Application of Research Results. The local static model is suitable for the study of the small-scale urban transportation network and can be used as the theoretical basis for the opening of closed residential communities in small cities. In the first stage of this paper, the operation of the local static model is simple, and the factors involved are relatively simple, including driving distance, driver response time, traffic delays at different intersections, and other factors. Due to the relatively simple traffic network in small-scale cities, the application of this model can make the calculation more accurate and efficient when studying the traffic conditions in small-scale cities, and finally quantify the improvement of road traffic capacity after the gated residential communities are opened, which makes the results more direct and clear.

The topological structure analysis model of complex road networks based on multilayer complex network coupling is suitable for traffic condition research of large complex road networks in first-tier and second-tier cities. From the perspective of global dynamics, the model comprehensively considers the influence of pedestrians and nonmotor vehicles on the traffic of motor vehicles under different road conditions at each network node, constructs a multi-layer complex network, and dynamically superimposes and couples it to obtain more accurate road data. This model improves the repeated calculation of traffic flow at intersections in previous studies and introduces factors such as pedestrians and nonmotor vehicles, resulting in more accurate conclusions than previous studies.

5.2. Effect Evaluation. The cost of congestion refers to the economic cost of citizens caused by congestion. According to relevant data from the National Bureau of Statistics, the cost of congestion per person per month in Beijing, Guangzhou, and Shenzhen has exceeded 700 yuan. Xiamen city was selected to verify the relevant conclusions in this paper, and the obtained conclusions have a high degree of stability with the actual situation. This part will also take Xiamen city as an example to carry out the corresponding calculation. According to the 2018 Annual Traffic Analysis Report of China's Major Cities jointly released by the Institute of Social Research of the Chinese Academy of Social Sciences and Amap, the average time of congestion in Xiamen is 25.43 minutes on a weekday. According to the data released by the Xiamen Municipal Bureau of Statistics, the average annual wage for urban on-private sector workers in Xiamen in 2018 was 83,839 yuan and the average monthly wage was $6,986.583$ yuan. The corresponding calculation is $6986.583 / 22 / 8 / 60=0.662$ yuan per minute ( 22 working days per month, 8 hours per day), then the time cost of congestion in Xiamen is 0.662 yuan per minute; in the end, each person lost $0.662 * 25.43 * 22,370=363$ yuan per month in commuting.

The opening of the community can improve the traffic capacity of the surrounding roads by $7 \%$, i.e., it reduces the congestion by about $7 \%$. This translates into an economic 


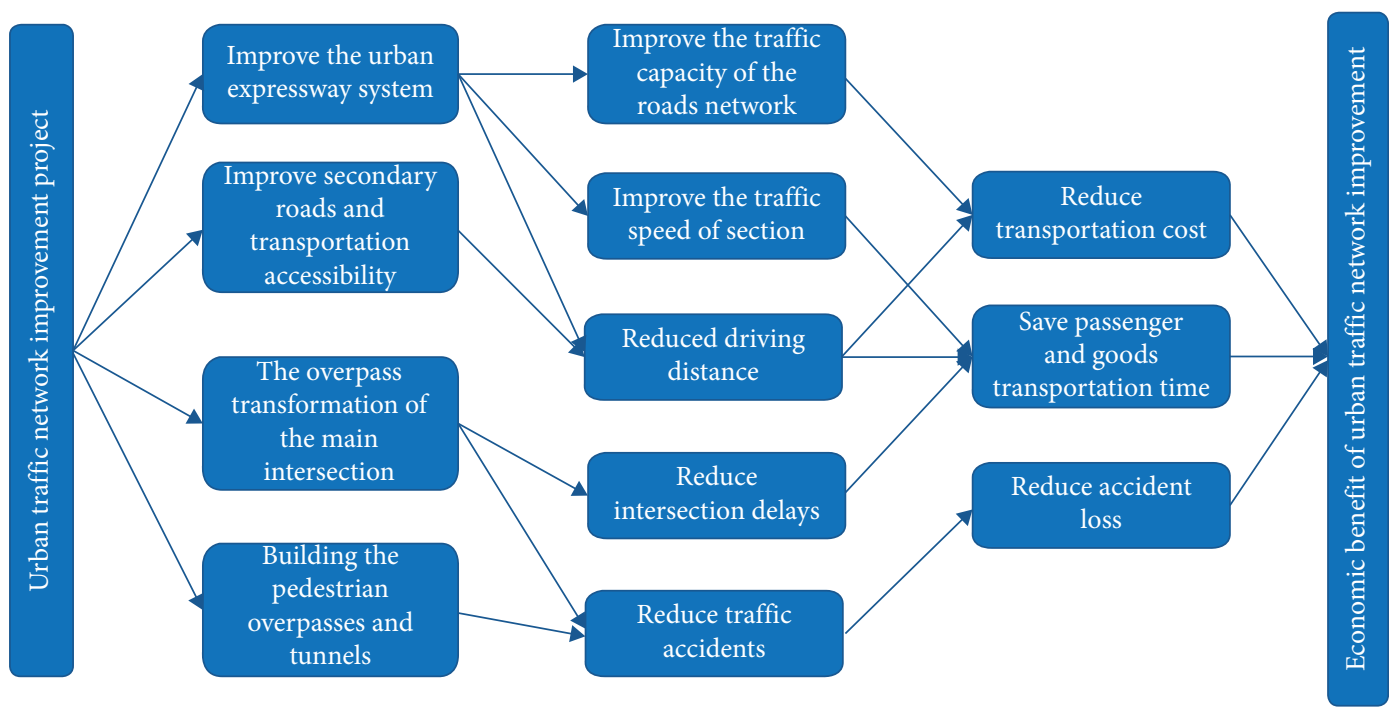

FIgURE 3: Causality diagram of road network improving economic benefits.

benefit of $370.363 * 5.7 \%=21.11$ yuan, which means that the congestion cost per person per month can be reduced by 21.11 yuan. Data from the Xiamen Municipal Bureau of Statistics show that Xiamen's population will reach 4.41 million in 2018, and the monthly congestion cost in Xiamen alone will reach 90 million yuan. In addition to reducing congestion costs, the improvement of road capacity will also have a great impact on the overall economy of the city.

As can be seen from Figure 3, the opening of gated residential communities can improve the traffic capacity of the urban road network, which can improve the operational speed, shorten the mileage, reduce delays, and reduce traffic accidents. Based on the method of economic cost-benefit analysis in "Methods and Parameters for Economic Evaluation of Highway Construction Projects," to calculate the project benefits, through qualitative analysis of the composition of unit vehicle transportation costs, the value of passenger travel time, and traffic accident losses, it can be seen that the improvement of urban road traffic capacity can reduce road operation costs and bring greater benefits to various commercial activities relying on the road transportation [23].

Apart from economic benefits, opening static gated residential communities will also bring in certain social benefits and environmental benefits, but the latter two are difficult to quantify, so the focus here is on economic benefits.

\section{Conclusions}

The opening of gated residential communities makes the regional road network more even, especially after the opening of large areas, as the integration degree and depth of urban roads are improved as a whole, and the attraction points and selectivity become more, which has a more obvious influence on the urban road network.

As far as road capacity is concerned, this paper describes the road capacity of isolated residential communities in a complete model from the perspective of local static state, and then, from the perspective of multilayer complex network coupling, taking residential areas as nodes, the mobile and nonmobile traffic unit solutions are formed into multilayer complex networks, and various topological parameters of the urban road network are further calculated, thus realizing dynamic coupling analysis of multilayer complex road network. The empirical research from RCA model with the local static state to multilayer complex network superposition coupling model with the global dynamic state is completed, and the conclusion is obtained that the community opening can improve the overall road capacity of the city by at least $11.3 \%$ by directly affecting the richness of the urban road network and the microcirculation system of urban roads.

At the same time, the improvement of the overall capacity of the road network in complex cities means that the losses caused by traffic congestion will be reduced. For some first-tier cities, the direct economic benefit of opening residential communities is as high as billion yuan per month. In addition to improving access efficiency, the role of shared parking spaces can be exploited, and the inclusion of time-of-day access and shared parking analysis can be considered in the future [20]. This change can promote the development of the local economy to some extent. Besides, the opening of the communities will also expand the scope of the original business circle and further promote the modernization of the city.

\section{Data Availability}

The data used to support the findings of this study are available from the corresponding author upon request.

\section{Conflicts of Interest}

The authors declare that they have no conflicts of interest.

\section{Acknowledgments}

The authors are very grateful to the National Social Science Foundation of China (Grant no. 17BJY076) for the support of their study. 


\section{References}

[1] National Bureau of Statistics of the People's Republic of China, China Statistical Yearbook, China Statistics Press, Beijing, China, 2019.

[2] F. Q. Zhao, "Influence on the structure of constructing quality urban road network on improving road capacity," Journal of Xi'an University (Natural Science Edition), vol. 21, no. 6, pp. 101-104, 2018.

[3] F. Yang, "Analysis of the status quo and development mode of small-scale industrial city traffic-taking Jinchang city, Gansu province as an example," Urban and Rural Construction, vol. 15 , pp. 57-59, 2020.

[4] M. T. Tang and Q. Zhang, "Optimization basis for road traffic planning in small towns in China," Urban Transportation, vol. 3, pp. 49-52, 2005.

[5] C. Y. Wu, M. B. Hu, R. Jiang et al., "Effects of road network structure on the performance of urban traffic systems," Physica A: Statistical Mechanics and Its Applications, vol. 563, 2021.

[6] Y. Cao, C. Wang, Y. M. Yang, J. T. Xu, and Y. E. Gao, "Multicause automatic real-time identification of urban road traffic congestion based on Bayesian network," Journal of Highway and Transportation Research and Development (English Edition), vol. 14, no. 4, 2020.

[7] J. Shi, Z. F. Li, and L. Tao, "Spatial distribution of residential communities and roadway planning," Urban Transport of China, vol. 9, no. 3, pp. 60-65, 2011.

[8] X. F. Wu, "Research of American new urbanism," M.S. thesis, East China Normal Univ., Shanghai, China, 2015.

[9] Y. H. Wang, "The coies in Chinese cites problems and countermeasures," Modern Urban Research, vol. 25, no. 3, pp. 85-90, 2010.

[10] J. Q. Shi, L. Cheng, Z. M. Chu, and Y. L. Liu, "Cellular automata model of urban road network traffic flow," Journal of Highway and Transportation Research and Development, vol. 32, no. 4, pp. 143-149, 2015.

[11] Y. Zhao, W. Du, and S. Chen, "Application of complex network theory to urban transportation network analysis," Urban Transport of China, vol. 7, no. 1, pp. 57-65, 2009.

[12] H. Q. Huang, "Research on the urban road short-term traffic flow forecast based on fuzzy theory," Highway Engineering, vol. 41, no. 1, pp. 172-175+192, 2016.

[13] L. L. Wang, J. Han, and Q. Wang, "Comprehensive analysis of impact of gated community on pedestrian transportation network, case study of Taipingqiao district in Shanghai," The Architect, vol. 3, pp. 24-31, 2015.

[14] X. D. Han, "The influence of the opening of gated communities in Jinan on the urban road network-a case study of five blocks in the main urban area," pp. 57-70, Chinese Society of Urban Planning, China, 2016.

[15] H. X. He and H. Liu, "Analysis on the rationality of opening closed communities," Higher Mathematics Research, vol. 21, no. 1, pp. 90-94, 2018.

[16] X. Y. Zheng and Y. X. Wang, "Opening vs. closing-a perspective on sustainable urban development," Modern Urban Studies, vol. 7, pp. 53-59, 2017.

[17] R. M. Li, C. Y. Jiang, F. H. Zhu, and X. L. Chen, "Traffic flow data forecasting based on interval type-2 fuzzy sets theory," IEEE/CAA Journal of Automatica Sinica, vol. 3, no. 2, pp. 141-148, 2016.

[18] Ministry of Housing and Urban-rural Development of the People's Republic of China, Standard for Urban
Comprehensive Transport System Planning, GB/T 51328-2018, Ministry of Housing and Urban-Rural Development, China, 2018.

[19] B.-J. He, D.-X. Zhao, J. Zhu, A. Darko, and Z.-H. Gou, "Promoting and implementing urban sustainability in China: an integration of sustainable initiatives at different urban scales," Habitat International, vol. 82, pp. 83-93, 2018.

[20] P. F. Wang, H. Z. Guan, P. Liu, P. F. Zhao, Y. Han, and H. Yue, "Allocation-pricing-revenue distribution mechanism of shared parking spaces," China Journal of Highways, vol. 33, no. 2, pp. 158-169, 2020.

[21] M. Dong and W. Q. Li, "The method of calculating the road capacity," Journal of Henan University (Natural Science), vol. 2, pp. 24-27, 2002.

[22] Ministry of Housing and Urban-rural Development of the People's Republic of China, Urban Residential Planning and Design Standards, GB50180-2018, Ministry of Housing and Urban-Rural Development, China, 2018.

[23] Y. B. Nie, "Study on the measuring and calculating method for economic benefits of urban transportation comprehensive improvement project," M.S. thesis, Changan University, Shaanxi Province, China, 2008. 